\title{
Assessing the effect of foreign travel and protection by BCG vaccination on the spread of tuberculosis in a low incidence country, United Kingdom, October 2008 to December 2009
}

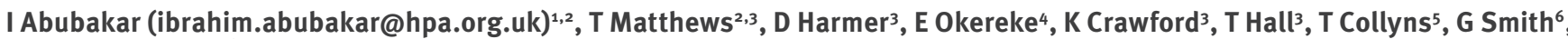 \\ A Barrett7, S Baugh ${ }^{8}$ \\ 1. Tuberculosis Section, Health Protection Agency Centre for Infections, Colindale, London, United Kingdom \\ 2. These authors contributed equally to the manuscript \\ 3. North Yorkshire and Humber Health Protection Unit, Hull, United Kingdom \\ 4. Health Protection Agency Yorkshire and Humber, Leeds, United Kingdom \\ 5. Leeds Teaching Hospitals Trust, St James’ University Hospital, Leeds, United Kingdom \\ 6. Health Protection Agency Regional Centre for Mycobacteriology, Birmingham, United Kingdom \\ 7. Health Protection Agency Regional Centre for Mycobacteriology, Newcastle, United Kingdom \\ 8. Northern Lincolnshire and Goole Hospitals National Health Service Foundation Trust, United Kingdom
}

Citation style for this article:

Citation style for this article: BCG vaccination on the spread of tuberculosis in a low incidence country, United Kingdom, October 2008 to December 2009 . Euro Surveill. 2011;16(12):pii=19826. Available online: http://www.eurosurveillance.org/ViewArticle.aspx?Articleld=19826

The contribution of travel to high incidence countries and the impact of the discontinuation of universal Bacillus Calmette-Guérin (BCG) vaccination to the recent rise in tuberculosis (TB) in the United Kingdom remain unclear. An outbreak in a college presented an opportunity to assess these. A cohort of students answered a questionnaire assessing risk factors for TB. Participants were screened with an interferon gamma release assay (IGRA). Unadjusted and adjusted odds ratios (OR) were calculated using logistic regression. Among 2,284 students, 400 (17.5\%) were diagnosed with TB infection. A higher risk was noted for travel to a high incidence area in the past two years (OR: 1.39; $95 \%$ confidence interval ( $\mathrm{Cl}): 1.04-1.89)$ and among those with the greatest exposure to the index case (OR: $3.94 ; 95 \% \mathrm{Cl}: 2.60-5.97)$. There was no association between BCG and risk of infection (OR: 1.05; 95\% $\mathrm{Cl}$ : 0.80-1.39). The lack of a protective effect by BCG on TB infection supports the discontinuation of universal vaccination. The association with foreign travel suggests the need to assess the cost-effectiveness of serial IGRA testing and treatment of positive persons among returning travellers.

\section{Introduction}

Countries with a low tuberculosis (TB) incidence, especially those heading towards elimination of the disease, have a policy of identifying individuals with presumed latent TB infection and offering prophylactic treatment $[1,2]$. In general, the policy prioritises the screening of recently exposed persons due to a higher risk of progression to active TB, often using a 'stone in the pond' approach [3]. This, together with the prompt diagnosis and adequate treatment of active cases, forms the cor- nerstone of TB control policy in countries with a low TB incidence.

The major burden of TB in many low-incidence highincome countries is in immigrants, their descendants and in particular high-risk groups such as the homeless, drug users and prisoners [4-6]. By contrast, for the rest of the population in these countries, the risk of TB remains low outside of outbreaks. The higher risk among the foreign-born is thought to be a function of infection prior to immigration and, to a lesser extent, travel to high TB incidence countries and transmission within immigrant communities. It is not clear however, to what extent each of these factors increases the risk of TB.

The Bacillus Calmette-Guérin (BCG) vaccine is the only preventive immunisation currently available against TB. Evidence suggests that it is effective in preventing meningitis and miliary TB in young children $[7,8]$. In the United Kingdom (UK), there is also evidence that BCG protects against pulmonary TB among school-aged children $[9,10]$. Most countries, including those with low TB incidence, have a BCG vaccination policy that may be universal or targeted. For low-incidence countries, a policy switch from universal to targeted BCG vaccination was supported by criteria put forward by the International Union Against Tuberculosis and Lung Disease in the 1990s [11]. Several countries with a low TB incidence, including the UK [12], have now changed their BCG vaccination policy to target high-risk groups. To date, the effect of this policy change has not been evaluated in the UK. In the United States (US), the poor efficacy of BCG following initial trials and its confounding effect on tuberculin skin testing led to the 
exclusion of BCG from the immunisation schedule [13]. The US guidelines allow the use of BCG in specific circumstances such as in children who cannot be removed from the source case. Arguably, if BCG is found to be efficacious against latent infection [14], and a person is travelling to a country with a high TB incidence and significant levels of anti-TB drug resistance, it may be justifiable to use the same rationale to offer BCG.

Following the diagnosis of sputum smear-positive cavitatory pulmonary TB in a college student, the local Health Protection Unit, working closely with the Respiratory Medicine Service and Primary Care Trust, screened 2,284 students and 299 staff members with an interferon gamma release assay (IGRA) and, where appropriate, chest X-ray examination. The students at the college were evenly divided in two groups: the age group vaccinated as part of the universal school-age BCG vaccination policy in the UK, and the group who reached the relevant age after the policy was changed. The investigation identified 19 cases of active TB. This outbreak presented an opportunity to assess the relative contribution of foreign birth, recent travel to a high incidence setting, community transmission (based on contact with a TB case and non-white ethnicity) and the protective effect of BCG vaccination.

We formed the hypotheses that, in addition to place of birth and exposure to the index case, travel to a high incidence country and BCG vaccination may predict latent infection as measured by a positive IGRA. The BCG policy change and a well-defined cohort exposed to a known infectious case allowed us to test these hypotheses.

\section{Methods \\ Participants}

The study population consisted of all students attending the college during daytime. All participants were aged over 16 years. Staff who directly taught these students were also screened. Between October 2008 and December 2009, students, friends of the index case and staff at the college were interviewed. It took a long time to complete the examination of all contacts because we used the 'stone in the pond' approach, where a small proportion of contacts were screened and further circles for testing were identified if the level of infection found was higher than expected, until eventually all exposed students were screened.

\section{Ascertainment of risk factors}

Interviews were used to collect information from all 2,284 students about:

- place of birth, and if born abroad, year of entry in the UK,

- previous contact with TB in the household,

- age,

- sex,

- ethnic group, broadly classified as Asian, White, Black, mixed or other,
- history of travel to a country outside western Europe, North America and Australasia in the preceding two years,

- history of BCG vaccination ascertained through the inspection of a scar or reliable recollection of vaccination,

- symptoms suggestive of TB.

The incidence of TB in the local area in 2007 was 4 per 100,000 with most cases occurring in non-White ethnic groups (T. Matthews, personal communication, Jan 2009). Based on this, we used non-White ethnicity as a marker of community risk of TB. At the same time a blood sample was drawn from each participant for IGRA testing. IGRA tests have been shown to have at least an equivalent level of diagnostic accuracy for latent TB infection compared with the tuberculin skin test (TST) [15]. Emerging evidence suggests that the predictive value of IGRAs for the development of active TB is also higher than $[16]$ or equivalent to TST $[17,18]$.

\section{Clinical and laboratory investigation}

Contacts were investigated in concentric circles of decreasing exposure in three groups based on exposure times over the 17 days that the index case was at the college while infectious. Group 1 was screened between November 2008 and January 2009 and included students who shared classrooms with the index case with cumulative exposures of more than two hours, or friends of the index case. Group 2 was screened in February 2009 and included students who attended the same general study class as the index case, with cumulative exposure time between one and two hours in a large hall setting. Group 3 was screened between July and August 2009 and included the rest of the college. Following the first two circles of screening, 270 students whose initial IGRA test had been negative or indeterminate were tested again.

Two IGRA tests were used, Quantiferon-TB Gold in tube test (QGIT, Cellestis) and T-Spot TB (Oxford Immunotec). For the majority of participants, the QGIT test was used. A small proportion of individuals were tested with T-Spot TB, and those with a negative result were retested using QGIT during the third circle of screening. Both IGRA tests use the region of difference-1 antigens early secretory antigen target 6 (ESAT-6) and culture filtrate protein 10 (CFP 10), and in addition QGIT includes TB7.7, to stimulate T-effector cells specific for Mycobacterium tuberculosis to produce gammainterferon. Those with a positive IGRA result from any screening round were recalled for a chest $\mathrm{X}$-ray examination and clinical review at a respiratory medicine clinic. Preventive therapy was offered to individuals younger than 35 years with a positive IGRA result but no evidence of active TB, according to national guidelines [1]. No formal ethical review was required as this was done as part of a service response. 


\section{TABLE 1}

Characteristics of the student population, tuberculosis college outbreak, United Kingdom, October 2008-

December $2009(\mathrm{n}=2,284)$

\begin{tabular}{|c|c|c|}
\hline Characteristic $^{a}$ & Category & n (\%) \\
\hline \multicolumn{3}{|l|}{ Demographic } \\
\hline \multicolumn{2}{|l|}{ Age, median (IQR) } & $17.8(17.3-18.5)$ \\
\hline \multirow{2}{*}{ Sex } & Male & $1,055(46.2)$ \\
\hline & Female & $1,229(53.8)$ \\
\hline \multirow{3}{*}{ Exposure } & Group $1^{\mathrm{b}}$ & $131(5.7)$ \\
\hline & Group 2 & $244(10.7)$ \\
\hline & Group 3 & $1,909(83.6)$ \\
\hline \multirow{2}{*}{ Household history } & Yes & $82(3.6)$ \\
\hline & No & $2,162(96.4)$ \\
\hline \multirow{2}{*}{ Place of birth } & UK & $2,089(91.6)$ \\
\hline & Not UK & $192(8.4)$ \\
\hline \multirow{2}{*}{ Travel in the last two years ${ }^{c}$} & Yes & $422(19)$ \\
\hline & No & $1,799(81)$ \\
\hline \multirow{5}{*}{ Ethnicity } & White & $2,061(90.2)$ \\
\hline & Asian & $170(7.4)$ \\
\hline & Black & $10(0.4)$ \\
\hline & Mixed & $29(1.27)$ \\
\hline & Other & $14(0.6)$ \\
\hline \multicolumn{3}{|l|}{ Clinical } \\
\hline \multirow{2}{*}{ BCG-vaccinated } & Yes & $1,067(49.6)$ \\
\hline & No & $1,082(50.4)$ \\
\hline \multirow{2}{*}{ IGRA } & Positive & $400(17.5)$ \\
\hline & Negative & $1,884(82.5)$ \\
\hline \multirow{2}{*}{ Chemoprophylaxis } & Yes & $349(15.7)$ \\
\hline & No & $1,912(84 \cdot 3)$ \\
\hline
\end{tabular}

BCG: Bacillus Calmette-Guérin; IGRA: interferon gamma release assay; IQR: inter-quartile range; UK: United Kingdom.

a For participants for whom information on the respective variable was available.

b This number is different from the Figure 2 as it only includes students (not friends).

Countries travelled to include Bangladesh, China, India, Russia and several countries in Sub-Saharan Africa.

\section{FIGURE 1}

Active tuberculosis cases, college outbreak, United

Kingdom, October 2008-December 2009 (n=20)

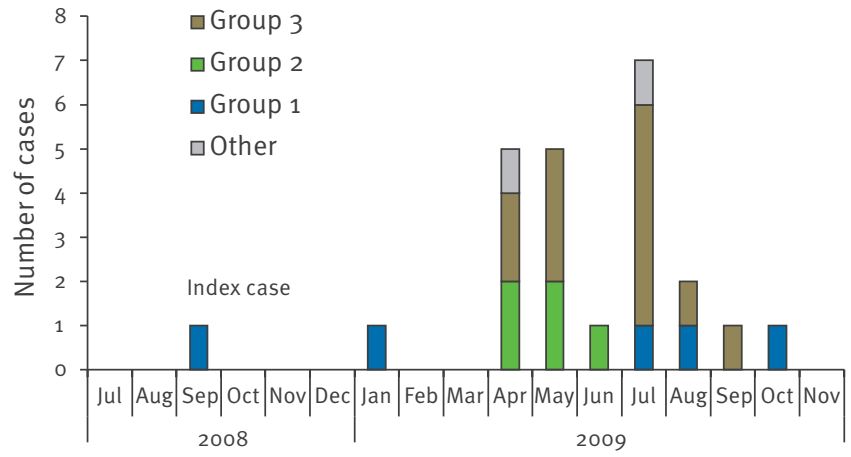

Group 1: cumulative exposure $\geq 2 \mathrm{~h}$ in classroom groups to the index case and friends of the index case. Numbers here differ from those in the Tables, where only students are taken into account .

Group 2: students exposed $1-2 \mathrm{~h}$ in a large hall setting.

Group 3: rest of the college. Other: screened in Groups 2 and 3 but diagnosed subsequently by their family doctor or local hospital.

\section{Definitions}

Participants were considered to have TB infection if they had a positive IGRA test or had active disease.

\section{Statistical methods}

Data were entered into a specifically designed database in Microsoft Access. Proportions were calculated for categorical variables and medians with inter-quartile ranges for continuous variables. We used univariable and multivariable logistic regression to investigate factors associated with a positive IGRA result as a proxy for latent infection. All variables considered a priori to be risk factors for TB were included in the model. We investigated the interaction between risk factors for TB using the likelihood ratio test. Data were analysed using the statistical software Stata 11.

\section{Results}

The median age of students was 17.8 years (interquartile range (IQR): 17.3-18.5 years) and 1,055 (46.2\%) were male (Table 1). Characteristics of the students are summarised in Table 1. Among the students, $49.6 \%$ were BCG-vaccinated, $90.2 \%$ were of white ethnicity, and $18.8 \%$ had travelled within the last two years to a high incidence country, including Bangladesh, China, India, Russia, and several countries in Sub-Saharan Africa.

\section{FIGURE 2}

Proportion of students with latent tuberculosis infection and active tuberculosis by screening circles, college outbreak, United Kingdom, October 2008-December 2009 $(\mathrm{n}=2,284)$

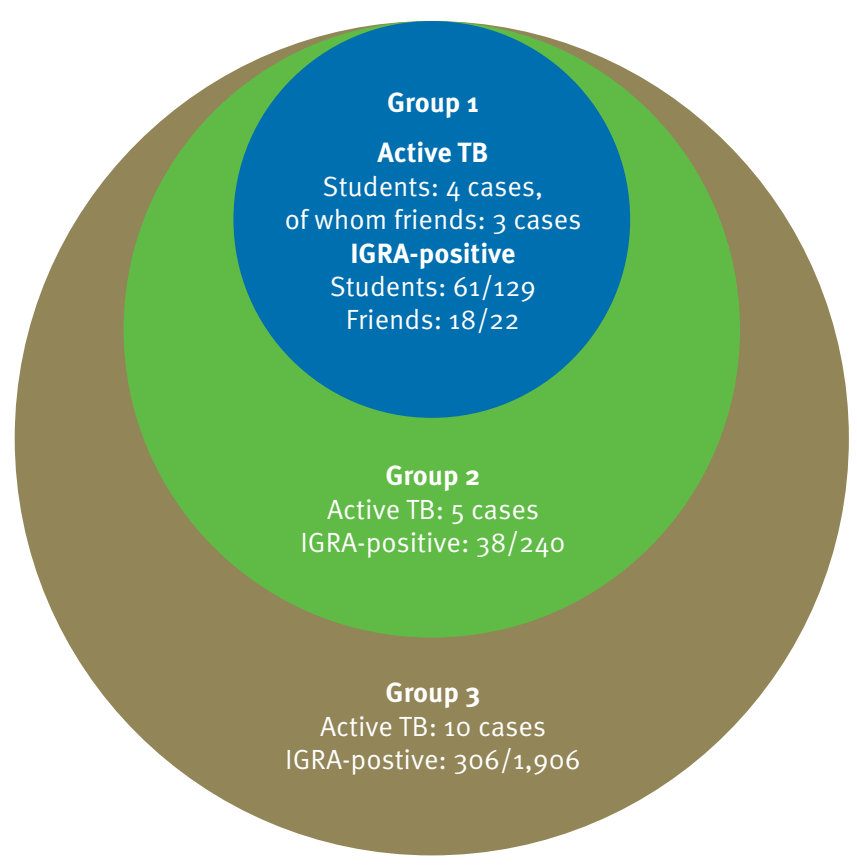

IGRA: interferon gamma release assay; TB: tuberculosis.

Group 1: cumulative exposure $\geq 2 \mathrm{~h}$ in classroom groups to the index case and friends of the index case. Nine of the 22 friends were also students. Numbers here differ from those in the tables, where only students are taken into account.

Group 2: students exposed 1-2h in a large hall setting. Group 3: rest of the college. 
The characteristics of the 19 active TB cases are described in detail elsewhere [19]. In brief, Figure 1 shows the distribution of cases over time with seven of the eight cases confirmed by bacterial culture sharing an identical 24 loci MIRU VNTR pattern (42234 2742511334422423255 ) with the index case.

The median age of all the staff was 46.8 years (IQR: 38.5 to 55.3 years), and 114 (38.1\%) were male. Of 299 staff members, $48(16.1 \%)$ had a positive IGRA test. No active TB cases were diagnosed among staff members.

\section{Characteristics of and factors associated} with TB infection among students

Of the 2,284 students screened, 400 (17.5\%) had evidence of TB infection. A further five friends had a positive IGRA test (shown in Figure 2) but were not included in this analysis as they were not students at the college. Figure 2 shows the number of individuals with a positive IGRA result including those with active TB in each circle of screening. The proportion of participants positive in the first, second and third circle of screening were $49.6 \%, 15.8 \%$ and $16.0 \%$ respectively. In addition, 11 of 270 students converted from an initial negative to a positive IGRA result in the third round of screening. The index case had 22 friends who were identified as close contacts, 16 of whom also attended the same college. Thirteen of those 16 were positive. Five of the six non-college friends had a positive IGRA result. All 400 students were offered preventive treatment according to national guidelines.
In the univariable analysis, being male and/or having a history of travel to a high-incidence country in the last two years were both positively associated with an increased risk of a positive IGRA result (Table 2 ). A sub-analysis conducted with only UK-born individuals did not change the outcome (data not shown). Being of white ethnicity and/or being in exposure Groups 2 or 3 were both associated with a lower risk of a positive IGRA result. In the fully adjusted model, being male (odds ratio (OR): 1.28; 95\% confidence interval (CI): $1.02-1.62 ; p=0.035$ ), being more exposed (exposure Group 1 compared to Group 3) (OR: 3.94; 95\% Cl: $2.60-$ 5.97), and having a history of travel to a high incidence country in the last two years (OR: 1.39 ; $95 \% \mathrm{Cl}: 1.04-$ 1.89; $\mathrm{p}=0.028$ ) remained associated with an increased risk of being infected at statistically significant level (Table 2). There was no evidence of a protective effect of BCG vaccination on TB infection (OR: 1.05 ; $95 \% \mathrm{Cl}$ : 0.80-1.39) and no statistically significant evidence of interaction between any of the risk factors.

\section{Discussion}

This study found a significant association between travel to high-incidence countries and the risk of latent TB infection among students. As expected, students in close contact with the index case were more likely to have evidence of latent infection. BCG vaccination did not have an effect on TB infection among the student population.

The level of latent infection found is higher than would be expected in a low incidence area. Although the incidence of TB has increased in the UK over the last two

\section{TABLE 2}

Risk factors for tuberculosis infection ${ }^{\mathrm{a}}$ among students, college outbreak, United Kingdom, October 2008-December 2009 $(\mathrm{n}=2,284)$

\begin{tabular}{|c|c|c|c|c|c|}
\hline \multicolumn{2}{|l|}{ Characteristic } & $n(\%)$ & $\begin{array}{l}\text { Univariable } \\
\text { OR }(95 \% \mathrm{Cl})\end{array}$ & $\begin{array}{c}\text { Multivariable } \\
\text { OR }(95 \% \mathrm{Cl})\end{array}$ & $p$ value $^{b}$ \\
\hline \multicolumn{2}{|l|}{ Age, median (IQR) } & $17(17-18)$ & $1.01(0.88-1.17)$ & $1.04(0.87-1.24)$ & 0.694 \\
\hline \multirow{2}{*}{ Sex } & Female & $192(15.8)$ & 1 & 1 & \\
\hline & Male & $209(19.9)$ & $1.32(1.06-1.64)$ & $1.28(1.02-1.62)$ & 0.035 \\
\hline \multirow{3}{*}{ Exposure } & Group 1 & $58(47.1)$ & $4.84(3.35-6.98)$ & $3.94(2.60-5.97)$ & \\
\hline & Group 2 & $38(15.8)$ & $0(0.13-0.36)$ & $0.84(0.55-1.29)$ & \\
\hline & Group 3 & $306(16.5)$ & 1 & 1 & 0.001 \\
\hline \multirow{2}{*}{ Household history } & No & $379(17.6)$ & 1 & 1 & \\
\hline & Yes & $16(19.5)$ & $1.13(0.65-1.98)$ & $1.41(0.79-2.53)$ & 0.247 \\
\hline \multirow{2}{*}{ Place of birth } & Not UK & $41(21.3)$ & 1 & 1 & \\
\hline & UK & $357(17.2)$ & $0.77(0.53-1.10)$ & $1.05(0.61-1.81)$ & 0.852 \\
\hline \multirow{2}{*}{ Travel in last 2 years } & No & $299(16.7)$ & 1 & 1 & \\
\hline & Yes & $91(21.6)$ & $1.38(1.06-1.79)$ & $1.39(1.04-1.89)$ & 0.028 \\
\hline \multirow{2}{*}{ Ethnicity } & Non-White & $51(22.9)$ & 1 & 1 & \\
\hline & White & $349(17.1)$ & $0.69(0.50-0.97)$ & $0.84(0.51-1.37)$ & 0.491 \\
\hline \multirow{2}{*}{ BCG-vaccinated } & No & $185(17.2)$ & 1 & 1 & \\
\hline & Yes & $186(17.5)$ & $1.02(0.81-1.28)$ & $1.05(0.80-1.39)$ & 0.723 \\
\hline
\end{tabular}

BCG: Bacillus Calmette-Guérin; CI: confidence Interval; IQR: interquartile range; OR: odds ratio; UK: United Kingdom.

a Tuberculosis infection includes all students with a positive IGRA and all students with active tuberculosis. The numbers therefore differ from the Table 1 which includes only IGRA positives.

b $\mathrm{p}$ values derived using likelihood ratio tests. 
decades [20], the rate of active TB identified in this college is several times higher than the rate for England in the equivalent age group. Indeed, in the preceding six years in the local Health Protection Unit area, only 10 people aged 16 to 20 years were diagnosed with TB. The proportion of students with latent infection in this college was similar to that observed in previously reported school outbreaks in the UK [21,22]. These other outbreaks occurred in schools with a high ethnic minority population where the high proportion with latent infection could, at least in part, be explained by a high background rate. This is unlikely in the setting described here as the proportion of ethnic minorities in the local population was very low. The low proportion of non-White ethnicity may also explain the lack of association with TB in the regression model. It is possible that in addition to the index case, other pulmonary TB cases within the school, possibly the smear-negative culture-positive cases, contributed to the transmission [23]. Figure 1 suggests this explanation is possible but does not prove it. An alternative explanation is that the index case was particularly infectious. Eighteen of 22 friends of the index case were infected, suggesting high infectiousness.

The significant association observed with travel to countries with a high incidence of TB, independent of exposure to the index case, provides an alternative explanation for the higher than expected rate of latent infection observed in the school. Previous studies have linked travel to high incidence countries with a higher risk of infection $[24,25]$. Based on these studies, Cobelens et al. [25] recommend that people travelling from low incidence countries to areas highly endemic for TB should be offered serial testing or BCG vaccination. To detect an effect, Cobelens et al. followed a cohort travelling to countries with very high TB endemicity with an annual risk of infection above $1 \%$ and found an increased risk associated with travel to high TB endemic countries. A previous descriptive UK study suggested a higher risk of active TB among individuals from the Indian sub-continent following return visits [26], although subsequent comparison with a control group found no association [27]. The study presented here provides the first evidence in the UK that travel to countries with high levels of TB infection may be an independent risk factor for acquiring latent TB infection. This effect was not mitigated by BCG vaccination. Unlike previous studies, we utilised IGRA tests not confounded by the majority of non-tuberculous mycobacteria or BCG vaccination. The observed increased risk is therefore likely to be a result of true latent TB infection acquired either during or prior to the current incident.

Acquisition of TB during foreign travel is particularly important because the incidence of multi- and extensively drug-resistant TB is higher in many parts of the world compared to the UK $[28,29]$. These strains may be acquired while travelling, with the potential for subsequent spread upon return to a low incidence country. An approach to reduce the incidence of travelacquired TB is to use serial TST before and after travel and treat those converting to a positive test for latent infection [25]. Unfortunately the acquisition of resistant strains would make this approach less effective, with limited options for the treatment of latent infection due to multi- and extensively drug-resistant TB [30]. Furthermore, some individuals will convert from a negative to a positive TST due to previous exposure to non-tuberculous mycobacteria.

That we did not observe protection by BCG against latent infection may reflect a true lack of effect against latent infection. It is widely accepted that BCG only protects against severe forms of TB in infants [31], however, emerging evidence suggests that it may also protect against latent infection [32,14]. As a result, alternative explanations for the absence of a protective BCG effect against latent infection among students have to be considered. As BCG immunisation policy in the UK includes the selective vaccination of infants at high risk, it is possible that vaccinated individuals were more likely to have been exposed to TB, leading to a failure to detect a protective effect. Analysis of our data, however, suggests that this is unlikely, as the observation was independent of the age at BCG vaccination. In addition, controlling for ethnic origin, the main criterion for selecting who to vaccinate, did not change the findings. Significant variation in the efficacy of BCG has been observed in different countries [8]. The reasons for variation, including exposure to non-tuberculous mycobacteria, latitude, use of different strains of the vaccine and genetic differences of the vaccinees have been discussed extensively [33] but do not provide an obvious explanation for the observed lack of effect in this study.

The retrospective collection of data on risk factors in our investigation limits the ability to infer a causal link with exposures such as travel history and BCG vaccination status due to the possibility of recall bias. For the majority of participants, we obtained TB exposure history and risk factor data at the time of initial screening, before the IGRA test results were known, and thereby minimised recall bias. Any bias would therefore have been non-differential reducing the probability of detecting a true effect. We did not collect baseline IGRA data, therefore some of the IGRA-positive persons may have acquired their latent infection remotely. Nevertheless, the high proportion of students with latent infection in a low-incidence area, the proportion of students who converted and the high proportion of cases of active TB with the same genotype suggest that we were observing the effects of recent transmission. As we identified, interviewed and tested the vast majority of the target population, our findings are not affected by non-response bias. Travel history, on the other hand, may have been biased because the exposure recorded over the preceding two years may merely reflect longer-term travel. However, this is unlikely to invalidate the observed association with travel. A 
further source of bias relates to the lack of vaccination cards to confirm the history of BCG vaccination. This is particularly important for children born abroad or from ethnic minority groups where scars may be less reliable due to vaccination at birth. Finally, although we used multivariable regression to adjust for the effect of several factors, residual confounding cannot be excluded.

The long time it took to screen the entire school possibly contributed to the emergence of secondary cases. While this may in part question the 'stone in the pond' approach, we contend that this incident is unusual. It is likely that controlling for exposure group accounted for the effect of secondary transmission in the analysis with infection as the outcome measure.

This study has found a significant association between travel to countries with a high TB incidence and the risk of latent TB infection among college students. The lack of a protective effect by BCG shows that an increased risk for TB infection during outbreaks may not be mitigated by a universal vaccination approach. This provides support for the discontinuation of universal BCG vaccination of children at school age. Travel appears to be an independent risk factor for the acquisition of TB infection. In the UK, BCG vaccination is currently recommended to people travelling to high-incidence countries for longer than three months. A cost-effectiveness analysis of the screening of travellers suggested that a single post-travel tuberculin skin test is the best approach [34]. However, this study did not assess the use of IGRAs. As IGRAs are not confounded by nontuberculous mycobacterial infection, future studies should evaluate the effectiveness and cost-effectiveness of a policy requiring serial IGRA testing of travellers and treatment of IGRA-positive persons upon their return from high-incidence countries.

\section{Acknowledgements}

Individuals too numerous to identify were involved in the management of this outbreak, including clinical and administrative support staff from the local Health Protection Unit, Primary Care Trust, acute hospital, local and regional laboratories, and staff and students of the college.

\section{Ethics and role of funding source}

The investigation was funded by the local National Health Service and the Health Protection Agency as part of the service response to the outbreak and therefore no ethical review was required. No external funding was received.

\section{References}

1. National Collaborating Centre for Chronic Conditions. Tuberculosis: clinical diagnosis and management of tuberculosis, and measures for its prevention and control. London: Royal College of Physicians; 2006. Available from: http://guidance.nice.org.uk/CG33/Guidance/pdf/English

2. Guidelines for the investigation of contacts of persons with infectious tuberculosis. Recommendations from the National Tuberculosis Controllers Association and CDC. MMWR Recomm Rep. 2005;54(RR-15):1-47.
3. Veen J. Microepidemics of tuberculosis: the stone-in-the-pond principle. Tuber Lung Dis. 1992;73(2):73-6.

4. Cain KP, Benoit SR, Winston CA, Mac Kenzie WR. Tuberculosis among foreign-born persons in the United States. JAMA. 2008;300(4):405-12.

5. Haddad MB, Wilson TW, ljaz K, Marks SM, Moore M. Tuberculosis and homelessness in the United States, 19942003. JAMA. 2005;293(22):2762-6.

6. Story A, Murad S, Roberts W, Verheyen M, Hayward AC. Tuberculosis in London: the importance of homelessness, problem drug use and prison. Thorax. 2007;62(8):667-71.

7. Trunz BB, Fine P, Dye C. Effect of BCG vaccination on childhood tuberculous meningitis and miliary tuberculosis worldwide: a meta-analysis and assessment of cost-effectiveness. Lancet. 2006;367(9517):1173-80.

8. Colditz GA, Berkey CS, Mosteller F, Brewer TF, Wilson ME, Burdick $E$, et al. The efficacy of bacillus Calmette-Guérin vaccination of newborns and infants in the prevention of tuberculosis: meta-analyses of the published literature. Pediatrics. 1995;96(1 Pt 1):29-35.

9. Sutherland I, Springett VH. Effectiveness of BCG vaccination in England and Wales in 1983. Tubercle. 1987;68(2):81-92.

10. Hart PD, Sutherland I. BCG and vole bacillus vaccines in the prevention of tuberculosis in adolescence and early adult life. Br Med J. 1977;2(6082):293-5.

11. Criteria for discontinuation of vaccination programmes using Bacille Calmette-Guerin (BCG) in countries with a low prevalence of tuberculosis. A statement of the International Union Against Tuberculosis and Lung Disease. Tuber Lung Dis. 1994;75(3):179-80.

12. Donaldson L, Beasley C, Smith J. PL CMO (2005)3: Changes to the BCG vaccination programme: London: Department of Health; 6 Jul 2005. Available from: http://www.dh.gov. uk/en/Publicationsandstatistics/Lettersandcirculars/ Professionalletters/Chiefmedicalofficerletters/DH_4114993

13. The role of BCG vaccine in the prevention and control of tuberculosis in the United States. A joint statement by the Advisory Council for the Elimination of Tuberculosis and the Advisory Committee on Immunization Practices. MMWR Recomm Rep. 1996;45(RR-4):1-18.

14. Soysal A, Millington KA, Bakir M, Dosanjh D, Aslan Y, Deeks J, et al. Effect of BCG vaccination on risk of Mycobacterium tuberculosis infection in children with household tuberculosis contact: a prospective community-based study. Lancet. 2005;366(9495):1443-51.

15. Pai M, Zwerling A, Menzies D. Systematic review: T-cell-based assays for the diagnosis of latent tuberculosis infection: an update. Ann Intern Med. 2008;149(3):177-84.

16. Diel R, Loddenkemper R, Meywald-Walter K, Niemann S, Nienhaus A. Predictive value of a whole blood IFN-gamma assay for the development of active tuberculosis disease after recent infection with Mycobacterium tuberculosis. Am J Respir Crit Care Med. 2008;177(10):1164-70.

17. Kik SV, Franken WPJ, Mensen M, Cobelens FGJ, Kamphorst $M$, Arend SM, et al. Predictive value for progression to tuberculosis by IGRA and TST in immigrant contacts. Eur Respir J. 2010;35(6):1346-53.

18. Hill PC, Jackson-Sillah DJ, Fox A, Brookes RH, de Jong BC, Lugos MD, et al. Incidence of tuberculosis and the predictive value of ELISPOT and Mantoux tests in Gambian case contacts. PLoS ONE. 2008;3(1):e1379.

19. Abubakar I, Matthews T, Harmer D, Okereke E, Crowford K, Hall $\mathrm{T}$, et al. Assessing an outbreak of tuberculosis in an English college population. Eur Respir J. Forthcoming.

20. Crofts JP, Gelb D, Andrews N, Delpech V, Watson JM, Abubakar I. Investigating tuberculosis trends in England. Public Health. 2008;122(12):1302-10.

21. Paranjothy S, Eisenhut M, Lilley M, Bracebridge S, Abubakar I, Mulla R, et al. Extensive transmission of Mycobacterium tuberculosis from 9 year old child with pulmonary tuberculosis and negative sputum smear. BMJ. 2008;337:a1184.

22. Ewer K, Deeks J, Alvarez L, Bryant G, Waller S, Andersen P, et al. Comparison of T-cell-based assay with tuberculin skin test for diagnosis of Mycobacterium tuberculosis infection in a school tuberculosis outbreak. Lancet. 2003;361(9364):1168-73.

23. Behr MA, Warren SA, Salamon H, Hopewell PC, Ponce de Leon $A$, Daley CL, et al. Transmission of Mycobacterium tuberculosis from patients smear-negative for acid-fast bacilli. Lancet. 1999;353(9151):444-9.

24. Cobelens FG, van Deutekom H, Draayer-Jansen IW, ScheppBeelen AC, van Gerven PJ, van Kessel RP, et al. Association of tuberculin sensitivity in Dutch adults with history of travel to areas of with a high incidence of tuberculosis. Clin Infect Dis. 2001;33(3):300-4. 
25. Cobelens FG, van Deutekom H, Draayer-Jansen IW, ScheppBeelen AC, van Gerven PJ, van Kessel RP, et al. Risk of infection with Mycobacterium tuberculosis in travellers to areas of high tuberculosis endemicity. Lancet. 2000;356(9228):461-5.

26. Ormerod LP, Green RM, Gray S. Are there still effects on Indian Subcontinent ethnic tuberculosis of return visits?: a longitudinal study 1978-97. J Infect. 2001;43(2):132-4.

27. Singh H, Joshi M, Ormerod LP. A case control study in the Indian subcontinent ethnic population on the effect of return visits and the subsequent development of tuberculosis. J Infect. 2006;52(6):440-2.

28. Abubakar I, Moore J, Drobniewski F, Kruijshaar M, Brown T, Yates $M$, et al. Extensively drug-resistant tuberculosis in the UK: 1995 to 2007. Thorax. 2009;64(6):512-5.

29. Wright A, Zignol M, Van Deun A, Falzon D, Gerdes SR, Feldman $\mathrm{K}$, et al. Epidemiology of antituberculosis drug resistance 2002-07: an updated analysis of the Global Project on Anti-Tuberculosis Drug Resistance Surveillance. Lancet. 2009;373(9678):1861-73.

30. Fraser A, Paul M, Attamna A, Leibovici L. Drugs for preventing tuberculosis in people at risk of multiple-drug-resistant pulmonary tuberculosis. Cochrane Database Syst Rev. 2006;(2). CD005435

31. Rodrigues LC, Diwan VK, Wheeler JG. Protective Effect of BCG against Tuberculous Meningitis and Miliary Tuberculosis: A Meta-Analysis. Int J Epidemiol. 1993;22(6):1154-8.

32. Eisenhut M, Paranjothy S, Abubakar I, Bracebridge S, Lilley M, Mulla R, et al. BCG vaccination reduces risk of infection with Mycobacterium tuberculosis as detected by gamma interferon release assay. Vaccine. 2009;27(44):6116-20.

33. Fine PE. Variation in protection by BCG: implications of and for heterologous immunity. Lancet. 1995;346(8986):1339-45.

34. Tan M, Menzies D, Schwartzman K. Tuberculosis screening of travelers to higher-incidence countries: a cost-effectiveness analysis. BMC Public Health. 2008;8:201 